\title{
Research on the construction of smart supply chain system under the background of supply side reform
}

\author{
Yonghui $\mathrm{CAO}^{1, \mathrm{a}}$, He JIANG ${ }^{* 1, \mathrm{~b}}$ \\ ${ }^{1}$ Department of Business Administration, Guangzhou College of Technology and Business, Guangzhou, China
}

\begin{abstract}
At present, China's economy has entered a new period of high-quality development from the stage of high-speed growth. The supply side structural reform focusing on removing production capacity, inventory, deleveraging, cost reduction and making up for weaknesses is a major strategic measure to enhance the driving force of sustained economic growth, solve the deep-seated problems of economic development and improve the quality of economic development. Accelerating the innovation and application of smart supply chain has become an important part of promoting supply side structural reform, cultivating new economic growth points and building a modern economic system. This paper mainly analyzes the emergence and research status of smart supply chain and the construction of smart supply chain system under the background of supply side reform, so as to promote the high-quality development of economy.
\end{abstract}

\section{Introduction}

At present, China's economy has entered a new period of high-quality development from the stage of rapid growth. Economic development faces the problems of insufficient effective demand, low quality and efficiency of supply side system. The supply side structural reform is an important strategic measure to enhance the driving force of sustained economic growth, solve the deep-seated problems of economic development, and improve the quality of economic development. Smart supply chain has significant effect on promoting supply side structural reform in the aspects of de stocking, reducing cost, solving supply-demand dislocation, and promoting industrial transformation and upgrading.

The Party Central Committee and the State Council attach great importance to the innovation and application of supply chain. State leaders have repeatedly proposed to promote supply chain optimization and innovation on different occasions. The report of the 19th National Congress of the Communist Party of China proposed: cultivate new growth points and form new driving forces in the fields of medium and high-end consumption, innovation leading, green and low-carbon, sharing economy, modern supply chain and human capital services. The guiding opinions on promoting the innovation and application of supply chain issued by the general office of the State Council puts forward: "by 2020, a smart supply chain system covering China's key industries will be basically formed, and about 100 leading global supply chain enterprises will be cultivated. China will become an important center for innovation and application of global supply chain." Accelerating the innovation and application of smart supply chain has become an important part of promoting supply side structural reform, cultivating new economic growth points and building a modern economic system. As shown in Figure 1.

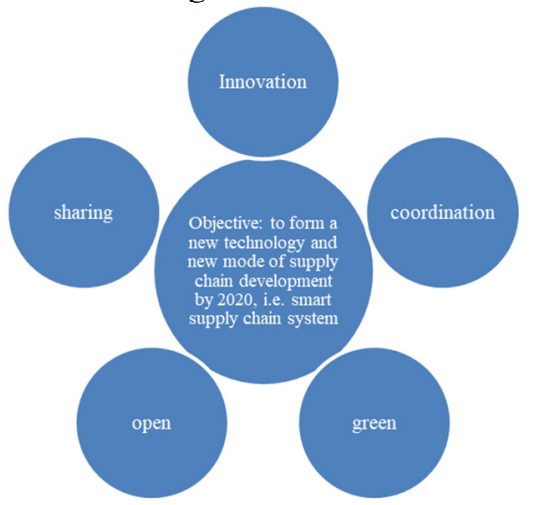

Fig1. construction concept of smart supply chain

\section{Literature research}

\section{1 generation and role of smart supply chain}

\subsubsection{First, the emergence of smart supply chain}

In 2009, IBM proposed a future oriented supply chain with three major characteristics: advanced, interconnected and intelligent. The concept of "smart logistics" is to generate real-time information through intelligent devices and systems. It is different from intelligent logistics. Intelligent logistics emphasizes the construction of a virtual logistics dynamic informatization of the Internet management system is different. "Smart logistics" pays more attention to the integration of the Internet of things, sensor network and the existing Internet. Through fine,

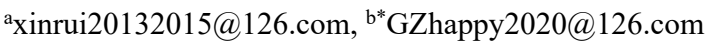


dynamic and scientific management, we can realize the automation, visualization, controllability, intelligence and networking of logistics, so as to improve the utilization rate of resources and the level of productivity, and create more comprehensive connotation of social value.

Luo Gang, a domestic scholar, put forward that "smart supply chain" is a comprehensive management integrated system built in and among enterprises by combining the Internet of things technology and the theory, method and technology of modern supply chain management. It can realize the intelligence, networking and automation of the supply chain. The core of smart supply chain is to make the members of the supply chain realize seamless connection in information flow, logistics, capital flow and so on. It tries its best to eliminate the influence of asymmetric information factors, and ultimately solves the problem of supply chain efficiency. Due to the embedded intelligent information network technology, compared with the traditional supply chain, the intelligent supply chain system has obvious advantages.

Although different scholars have different definitions of smart supply chain, we can summarize the following aspects: first, the smart supply chain under the "Internet plus" can enable enterprises to track and understand the real demand information and state of customers in real time by using intelligent information system, and provide more personalized services. Secondly, with the help of Internet, Internet of things, cloud computing and other innovative technologies, the whole process of smart supply chain can be visualized, so that enterprises can timely understand the status and activities of each link, and effectively control the overall operation process of the supply chain. Thirdly, smart supply chain is a flexible organization with strong process processing and intelligent response capabilities. It can make use of the main bodies to establish their own core competitiveness of the supply chain, integrate the supply chain, and closely connect the planning and execution in real time, so as to further realize its service function. Finally, the smart supply chain has a good early warning system. It can monitor the whole process, prevent supply chain interruption and other risks, and achieve the combination of intelligent agility and high-efficiency lean in the supply chain, and realize precision sensitivity.

\subsubsection{Second, the value of smart supply chain}

Smart supply chain has important value, which can be analyzed from the following three levels:

From the micro level, smart supply chain based on information technology can optimize business processes, improve market response speed, reduce enterprise costs, make the supply chain transparent, flexible and agile, and make all functions more collaborative. It is the core source for enterprises to obtain competitive advantage.

From the industrial level, smart supply chain is an important starting point to promote the supply side structural reform, which is conducive to reducing costs and increasing efficiency, better connecting production and consumption, and promoting supply-demand matching and consumption upgrading.
From the national level, the world economy has entered the era of supply chain competition from enterprise competition. The United States has upgraded the supply chain strategy to the national strategy. The level of supply chain, especially the development of smart supply chain, has become one of the important signs to measure a country's comprehensive strength.

\section{2 characteristics of smart supply chain}

Compared with the traditional supply chain, smart supply chain has more market elements, technical elements and service elements, showing five significant characteristics: first, systematic. It pays attention to the system optimization and the performance of the whole supply chain, and emphasizes "leading one to move the whole body". Second, synergy. It can form demand plan through demand perception, focus on vertical process end-to-end integration, and form intelligent supply chain on this basis. Third, accuracy and effectiveness. It promotes the iterative upgrading of products and services. Fourth, comprehensiveness. It involves product life cycle, market, supplier, factory building, process, information and other elements. Fifth, lean. It attaches importance to the lean manufacturing based on the whole value chain, from lean production to promoting lean logistics, lean procurement and lean distribution.

\section{3 research status of smart supply chain}

Smart supply chain is still a new theoretical concept. The research on smart supply chain is still in its infancy, mainly from the following two aspects:

\subsubsection{First, the construction of smart supply chain system under the background of informatization}

Li et al. constructs a distributed node deployment model of coastal port intelligent logistics supply chain under cloud computing. The simulation results show that the model can improve the scheduling path optimization and intelligent control ability of coastal port intelligent logistics supply chain. Zhang Liyuan established commodity archives in the manufacturing process, realized automatic shelf loading and intelligent flow sorting in the distribution link, and realized the application of automatic receiving and hand-held terminal inventory in the retail link through RFID technology, and proposed a smart supply chain solution. All the information of the whole supply chain is under the tracking and monitoring of the intelligent supply chain system, so as to realize the efficient operation of the supply chain[1]. Zhang Yuchuan and Wang Haiyan proposed a smart supply chain framework model based on the development of smart city and Internet of things, combined with the current situation of small and medium-sized enterprises in the supply chain[2]. Wang He proposed the construction idea of intelligent supply chain platform based on the internal relationship between the Internet of things and the supply chain[3]. Qiu Fusheng proposed to build an intelligent supply chain from the aspects of intelligent strategy, 
intelligent supply chain platform and supply chain early warning, aiming at the problems in the supply chain management process of manufacturing enterprises[4]. Huang Chengcheng et al. mainly constructed the intelligent supply chain system from the three modules of business, technology and management[5].

\subsubsection{Second, the application of smart supply chain in the industry}

Since the rise of smart supply chain, the related research is increasingly rich. Many research literatures are based on the supply chain of a specific field, which provides future research directions for different fields. In the agricultural supply chain, most of the existing researches rely on emerging technologies to combine agriculture with science. Through the construction of smart agricultural supply chain, we can better adapt to the era of transformation from traditional farming civilization to Internet civilization. For example, Xue Nan et al. proposed to establish an intelligent supply chain platform for agricultural products from the perspective of the Internet of things in view of the high cost of production and circulation links, confusion of standard system and many other problems in the Beijing Tianjin Hebei integrated agricultural product supply chain[6].

In the e-commerce industry, most of the existing studies focus on the development mode of e-commerce giants, and discuss the development mode that can be promoted. For example, Liu Jieqiong studied how Jingdong builds a smart supply chain in the era of intelligence. Through the introduction of Jingdong smart supply chain, this paper analyzes the core competitiveness of Jingdong smart supply chain, and puts forward the challenges faced by Jingdong in developing smart supply chain strategy[7].

In the aspect of supply chain finance, scholars mainly study the broad prospects of smart supply chain finance. For example, He Haifeng studied the mode innovation and case analysis of smart supply chain finance, summarized ten major models of Internet supply chain finance, and proposed the necessity of building a comprehensive enterprise financial service platform.

In addition, some scholars think that in the process of supply chain management, the fourth party logistics enterprises mainly have the problem of decentralized integration of multi-party information in the supply chain. This has a great impact on the development of the fourth party logistics enterprises. Therefore, they proposed to combine the supply chain management theory and intelligent information technology to build an intelligent supply chain model, which can solve the problems of the fourth party logistics enterprises. Some scholars use the goal programming theory to construct the cost house control system, and control and optimize the supply chain cost through the multi-level programming model.

\section{Construction of smart supply chain system under the background of supply side reform}

In recent years, with the continuous development of supply chain and the close relationship between upstream and downstream enterprises, Chinese enterprises have realized the role of supply chain management in promoting the development of enterprises. With the development of modern information technology, Chinese enterprises further strengthen the investment in supply chain management. Many intelligent information systems and decision support systems have emerged. Enterprises began to enter the stage of intelligent supply chain. However, at present, the performance of smart supply chain of Chinese enterprises is still at a low level, especially in the logistics industry, agriculture and fastselling products industry. Therefore, it is necessary to strengthen the support for the development of smart supply chain from the aspects of policy, capital and talents. By establishing a scientific and reasonable performance evaluation mechanism, we can further build and develop the smart supply chain. At the same time, it needs to give full play to the leading role of core enterprises. Through seeking strategic partners, strengthening logistics management, strengthening the application of modern information technology, and then establishing information sharing mechanism and customer management system, the advantages of smart supply chain can be fully displayed, the efficiency of China's industrial chain can be improved, and the market influence of enterprises can be enhanced.

\subsection{Improve the understanding of smart supply chain and strengthen the supply chain strategy}

Compared with developed countries, the construction of supply chain system in China's manufacturing industry is still in the exploratory stage, and the foundation is weak. At the same time, the majority of enterprises do not have a deep understanding of the nature of the supply chain, nor do they know how to implement it, how to cut in and operate from the perspective of intelligent supply chain. There is no smart supply chain strategy and no clear value direction guidance, which makes the manufacturing enterprises in China face many difficulties when facing intelligent manufacturing. Therefore, manufacturing enterprises need to deepen the understanding of smart supply chain. By formulating the development strategy of smart supply chain, the development direction of personalized supply chain is clear, such as the level of intelligence, the response level of customer service, the efficiency of product circulation, etc. Under the guidance of smart supply chain strategy, we will lead the enterprise production to intelligent iterative upgrading, and ensure the realization of enterprise operation and development goals. 


\subsection{The government should do a good job in top- level design, encourage and guide enterprises to implement smart supply chain}

The government attaches great importance to the important role of smart supply chain in boosting the real economy, and guides and supports enterprises to explore and practice smart supply chain. We should cultivate smart supply chain to serve leading enterprises. Through the implementation of the pilot demonstration project of smart supply chain enterprises by the government, we can promote the innovative application of smart supply chain in agriculture, circulation, foreign trade and other key industries. Smart supply chain enterprises should be encouraged to build open and shared supply chain management information platform. By guiding and promoting the information sharing of each node enterprise in the supply chain, the important role of intelligent supply chain in improving enterprise efficiency, promoting industrial transformation and upgrading, and improving the quality of economic development are fully released.

\section{3 strengthen the construction of smart supply chain data interconnection and build a smart supply chain platform}

Smart supply chain is a new type of enterprise organization based on technology, which needs the cooperation of upstream and downstream enterprises. At present, the degree of enterprise informatization is different. There are various standards for data acquisition and storage. The limitation of technology application and the lack of data standards result in the difficulty of data connection among enterprises, which affects the information interconnection and supply chain efficiency. It is suggested to speed up the development and promotion of key standards for big data. We should establish and constantly improve the big data standard system covering basic, data, technology, management and security, and promote information interconnection and sharing among enterprises.

The construction of smart supply chain is inseparable from the collaborative interaction between upstream and downstream enterprises. At present, enterprises should integrate information computing and manufacturing technologies such as Internet of things and cloud computing to build a smart supply chain platform. We should realize the whole system, whole life cycle and allround linkage with the software and hardware manufacturing resources of upstream and downstream enterprises, and then realize the integration and sharing of human, machine, material and information, and finally form the intelligent supply chain ecosystem.

\section{4 strengthen the construction of professional talent support system of smart supply chain}

The core of smart supply chain is supply chain technology and supply chain talents. Government encourages supply chain enterprises to set up training institutes to support their technological progress and innovation and application of smart supply chain. However, China's enterprises have a low level of informatization, lack of technology and professionals, so it is difficult to truly understand, attach importance to and use the smart supply chain, which will affect the coordinated development of smart supply chain. It is suggested to integrate university, enterprise and social resources, and establish a multi-level and multi type supply chain talent training and evaluation system. The government should support universities and enterprises to establish internship training mechanism, strengthen the training of practical skills of supply chain talents, and actively cultivate innovative talents of supply chain technology and application. Relying on enterprise and social resources, training institutions actively carry out supply chain knowledge popularization, education and training, and improve the overall cognition and application level of enterprises. Through the improvement of supporting measures, we can cultivate leading talents in the field of smart supply chain, so as to attract international supply chain talents to innovate and start businesses.

\section{5 establish a scientific and reasonable performance evaluation mechanism of smart supply chain}

Scientific and reasonable performance evaluation mechanism can supervise the supply chain, find problems and improve it. The research on smart supply chain management is just emerging in China. At present, there is still a lack of reasonable and effective evaluation mechanism for smart supply chain management. As a result, the management of enterprises is backward and the advantages of intelligent supply chain are not fully utilized. Scientific and reasonable performance evaluation model of smart supply chain can help enterprises find problems, reduce management costs, broaden the scope of cooperation, and truly realize supply chain competition. Therefore, it is necessary to establish a scientific and reasonable performance evaluation model of smart supply chain, so that enterprises can clearly and accurately judge their own smart supply chain management level, and then continuously promote the improvement of China's supply chain management level.

\section{Conclusion}

With the wave of the fourth scientific and technological revolution, the development of global supply chain has entered the era of intelligent supply chain. Smart supply chain is based on Logistics Internet and logistics big data. It is guided by enhancing customer value, and through collaborative sharing, innovation mode and advanced artificial intelligence technology, it realizes the organization form of efficient collaboration in the whole process of product design, procurement, production, sales and service. The characteristics of "wisdom" are embodied in the application of modern intelligent technology and supply chain technology. The whole operation of supply chain realizes the functions of visualization, perception and adjustment. Through flexible management, rapid 
response and intelligent collaboration, smart supply chain can achieve the development goals of innovation, ecology and efficiency of supply chain, so as to promote highquality economic development in the supply side reform.

\section{Acknowledgment}

This work is financially supported by outstanding scholars of philosophy and Social Sciences in Henan Province higher education in 2018, 2018-YXXZ-06; Henan philosophy and Social Sciences Planning Project in 2019, 2019BJJ003; The social development project of Henan provincial key R \& D and promotion project (Science and technology tackling key problems) in 2019, project number: 515; The science and technology innovation talent support program project of Henan Province higher education (HUMANITIES AND SOCIAL SCIENCES) in 2017, 2017-cxrc-004; Foshan social science planning project in 2020, 2020-QN11. Henan philosophy and Social Sciences Planning Project in 2019, 2019BJJ032. Thanks for the help.

\section{References}

1. Zhang Liyuan. Smart supply chain solutions based on RFID technology [J]. China integrated circuit, 2013,04:16-19.

2. Zhang Yuchuan, Wang Haiyan. Research on smart supply chain framework of small and medium-sized enterprises based on Internet of things [C]. China Management Modernization Research Association, Fudan Management Award Foundation, 2012, 7.

3. Wang he. Research on Kehui supply chain based on Internet of things technology [J]. China management informatization, 2015, 18 (14), 91-92.

4. Qiu Fusheng. Construction of intelligent supply chain for intelligent manufacturing $[\mathrm{J}]$. Logistics technology and application, 2017, 22 (3): 92 - 94, 96 -98 .

5. Huang Chengcheng, ye chunsen, Wang xuexuan, et al. Construction of smart supply chain system [J]. Value engineering, 2018, 37 (23): 121-123.

6. Xue Nan, Jiang Xi. Construction of intelligent supply chain of Beijing Tianjin Hebei integrated agricultural products based on Internet $10[\mathrm{~J}]$. China circulation economy, 2015,07:82-87.

7. Liu Jieqiong. Thinking on Jingdong's efforts to build a smart supply chain with the advent of intelligent era [J]. Science and technology innovation guide, 2018, 28 (137), 137-139. 